600 W. Grothe: Einwirk. v. Kaliumsulf hydrat etc. auf Chloracetylanilide

Berechnet

auf die Formel $\mathrm{C}_{8} \mathrm{H}_{10} \mathrm{SO}_{2}$ :

$\mathrm{C}_{8}=96=56,48 \%$

$\mathrm{H}_{10}=10=5,88$ "

$\mathrm{S}=32=18,82$ "

$\mathrm{O}_{2}=32=18,82$,
Gefunden:

I.

II.

$56,37 \%$

5,91 ,

$19,09 \%$

\title{
Ueber die Einwirkung von Kaliumsulfhydrat, Kaliumcyanid und Kaliumrhodanid auf Chlor- acetylanilide.
}

Von W. Grothe.

\section{Einwirkung von alkoholischem Kaliumsulfhydrat auf die chloracetylierten Anilide.}

Da in den Chloracetylverbindungen das Chloratom sehr leicht beweglich schien, so wurde auch der Austausch gegen andere Gruppen versucht. Als zu diesem Zwecke die Chloracetylverbindungen mit alkoholischer Kaliumsulfhydratlösung behandelt wurden, trat nicht, wie zu erwarten war, der Ersatz des Chloratoms durch die Gruppe SH, also Bildung von Derivaten der Thioglykolsäure ein, sondern in allen Fällen wurden unter Entwickelung von $\mathrm{H}_{2} \mathrm{~S}$ nur Sulfide erhalten. Der Prozess verlief glatt im Sinne folgender Gleichung:

$2 \mathrm{R} \cdot \mathrm{NH} \cdot \mathrm{CO} \cdot \mathrm{CH}_{2} \mathrm{Cl}+2 \mathrm{KSH}=\left(\mathrm{R} \cdot \mathrm{NH} \cdot \mathrm{CO} \cdot \mathrm{CH}_{2}\right)_{2} \cdot \mathrm{S}+2 \mathrm{KCl}+\mathrm{H}_{2} \mathrm{~S}$.

Die gebildeten Verbindungen sind aufzufassen als Derivate der Thiodiglykolsäure $\mathrm{S}<\mathrm{CH}_{2} \mathrm{COOH}$, in welcher das Hydroxyl der beiden Karboxylgruppen durch Reste des betreffenden Amidokörpers ersetzt ist. Die Reaktion wurde in folgender Weise ausgeführt.

Trägt man in die alkoholische Lösung der Chloracetylverbindung nach und nach alkoholische Kaliumsulfhydratlösung in geringem Ueberschuss ein, so zeigt schon in der Kälte eine Abscheidung von Chlorkalium und der Geruch nach Schwefelwasserstoff den Beginn der Reaktion an. Dieselbe wurde durch etwa halbstündiges Erhitzen im Wasserbade zu Ende geführt und hierbei eine lebhafte Entwickelung von Schwefelwasserstoffgas beobachtet. Beim Erkalten scheidet sich das in den meisten Lösungsmitteln schwer lösliche Reaktionsprodukt $a b$, indem die ganze Mischung zu einem Krystallbrei erstarrt. Der gebildete Körper wird dann durch Auswaschen vom überschüssigen Kaliumsulfhydrat und dem beigemengten Chlorkalium befreit und durch Umkrystallisieren rein gewonnen. 
W. Grothe: Einwirk, v. Kaliumsulf hydrat etc, auf Chloracetylanilide. 601

Thiodiglykolsäuredianilid

$\left(\mathrm{C}_{6} \mathrm{H}_{5} \mathrm{NH} \cdot \mathrm{CO} \mathrm{CH}_{2}\right)_{2} \cdot \mathrm{S}$.

Bei der Einwirkung von Kaliumsulfhydrat auf Chloracetanilid erhält man die Verbindung durch Umkrystallisieren aus Alkohol in langen, weissen, sehr leichten Nadeln, die bei $169^{\circ}$ schmelzen. Sie sind unlöslich in Wasser, schwer löslich in Alkohol, Aether, Benzol, Essigester.

\section{Analysen:}

I. $0,1938 \mathrm{~g}$ Substanz gaben $0,456 \mathrm{~g} \mathrm{CO}_{2}=0,1243 \mathrm{~g} \mathrm{C}=64,11 \% \mathrm{C}$ und $0,0979 \mathrm{~g} \mathrm{H}_{2} \mathrm{O}=0,01087 \mathrm{~g} \mathrm{H}=5,61 \% \mathrm{H}$.

II. $0,222 \mathrm{~g}$ Substanz gaben $0,1722 \mathrm{~g} \mathrm{Ba} \mathrm{SO}_{4}=0,02365 \mathrm{~g} \mathrm{~S}=10,65 \% \mathrm{~S}$.

Berechnet auf die Formel $\mathrm{C}_{18} \mathrm{H}_{10} \mathrm{~N}_{2} \mathrm{SO}_{2}$ :

$$
\begin{aligned}
& \mathrm{C}_{16}=192=64,00 \% \\
& \mathrm{H}_{16}=16=5,33 \% \\
& \mathrm{~N}_{2}=28=9,35 \% \\
& \mathrm{O}_{2}=32=10,66 \% \\
& \mathrm{~S}=32=10,66 \% \\
& \hline 300 \quad 100,00 \% .
\end{aligned}
$$

\section{Gefunden:}

I.

II.

Die Verbindung ist identisch mit dem von Anschütz ${ }^{1}$ ) und Biernaux durch Einwirkung von Thiodiglykolsäurechlorid auf Anilin gewonnenen Produkte, für welches die Verfasser den Schmelzpunkt $168^{\circ}$ angaben.

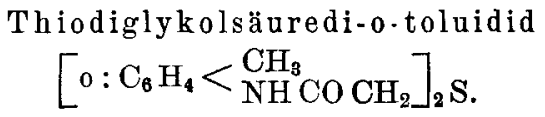

Aus verdünntem Eisessig krystallisiert die Verbindung in weissen, warzenförmig angeordneten Krystallnadeln vom Schmelzpunkt $190^{\circ}$. Dieselben sind unlöslich in Wasser, schwer löslich in Alkohol und Methylalkohol, leichter in heissem Eisessig, Benzol, Essigäther.

\section{Analysen:}

I. $0,1687 \mathrm{~g}$ Substanz gaben $0,406 \mathrm{~g} \mathrm{CO}=0,1107 \mathrm{~g} \mathrm{C}=65,63 \% \mathrm{C}$ und $0,0972 \mathrm{~g} \mathrm{H}_{\mathbf{2}} \mathrm{O}=0,0108 \mathrm{~g} \mathrm{H}=6,41 \% \mathrm{H}$.

II. $0,2961 \mathrm{~g}$ Substanz gaben $0,2133 \mathrm{~g} \mathrm{Ba} \mathrm{SO}_{4}=0,02929 \mathrm{~g} \mathrm{~S}=9,89 \% \mathrm{~S}$.

Berechnet auf die Formel $\mathrm{C}_{18} \mathrm{H}_{20} \mathrm{~N}_{2} \mathrm{SO}_{2}$ :

$$
\begin{aligned}
& \mathrm{C}_{18}=216=65,85 \% \\
& \mathrm{H}_{20}=20=6,09{ }^{\prime} \\
& \mathrm{N}_{2}=28=8,56 \% \\
& \mathrm{O}_{2}=32=9,75 \% \\
& \mathrm{~S}=32=9,75, \\
& \hline 328 \quad 100,00 \% .
\end{aligned}
$$

1) Annal. 273, 71 .

\section{Gefunden:}

I. $65,63 \%$

II.

6,41

4 
Thiodiglykolsäuredi-p-toluidid

$$
\left[\mathrm{p}: \mathrm{C}_{6} \mathrm{H}_{4}<\underset{\mathrm{NHCO}}{\mathrm{CH}_{3}} \mathrm{CH}_{2}\right]_{2} \mathrm{~S} \text {. }
$$

Aus schwach verdünntem Eisessig krystallisiert der Körper in feinen, weissen, bei $194^{\circ}$ schmelzenden Nädelchen, die in Wasser unlöslich, auch in Aether und Alkohol wenig löslich, leichter aber in warmem Eisessig und Essigester löslich sind.

\section{Analysen:}

I. $0,1768 \mathrm{~g}$ Substanz gaben $0,426 \mathrm{~g} \mathrm{CO}_{2}=0,1161 \mathrm{~g} \mathrm{C}=65,71 \% \mathrm{C}$ und $0,1002 \mathrm{~g} \mathrm{H}_{2} \mathrm{O}=0,01113 \mathrm{~g} \mathrm{H}=6,29 \% \mathrm{H}$.

II. $0,2288 \mathrm{~g}$ Substanz gaben $0,1645 \mathrm{~g} \mathrm{Ba} \mathrm{SO}_{4}=0,0226 \mathrm{~g} \mathrm{~S}=9,87 \% \mathrm{~S}$.

III. $0,1708 \mathrm{~g}$ Substanz gaben bei $18^{\circ}$ und $746 \mathrm{~mm}$ Barometerstand $12,8 \mathrm{ccm}$ Stickstoff $=8,49 \% \mathrm{~N}$.

Nach den Berechnungen bei der entsprechenden 0 :Verbindung enthält die unitäre Formel $\mathrm{C}_{18} \mathrm{H}_{20} \mathrm{~S} \mathrm{~N}_{2} \mathrm{O}_{2} 65,85 \% \mathrm{C}, 6,09 \% \mathrm{H}, 9,75 \% \mathrm{~S}$ und $8,56 \% \mathrm{~N}$.

Die Analysenwerte waren: I. $65,71 \% \mathrm{C}$ und $6,29 \% \mathrm{H}$; II. $9,87 \% \mathrm{~S}$; III. $8,49 \% \mathrm{~N}$.

$$
\begin{aligned}
& \text { Thiodiglykolsäuredi-0-xylidid } \\
& {\left[\mathrm{o}(a): \mathrm{C}_{6} \mathrm{H}_{3}<\mathrm{NH} \cdot\left(\mathrm{CH}_{3}\right)_{2} \mathrm{CO} \mathrm{CH}_{2}\right]_{2} \cdot \mathrm{S} \text {. }}
\end{aligned}
$$

Aus heissem Alkohol rein erhalten, bildet es feine, weisse, verfilzte, leichte Nadeln vom Schmelzpunkt 194\%. Löslich in Alkohol, Essigester, Eisessig, Benzol, unlöslich in Wasser.

Analysen:

I. $0,1838 \mathrm{~g}$ Substanz gaben $0,4525 \mathrm{~g} \mathrm{CO}_{2}=0,1234 \mathrm{~g} \mathrm{C}=67,14 \% \mathrm{C}$ und $0,1136 \mathrm{~g} \mathrm{H}_{\mathbf{8}} \mathrm{O}=0,0126 \mathrm{~g} \mathrm{H}=6,81 \% \mathrm{H}$.

II. 0,2536 g Substanz gaben $0,1684 \mathrm{~g} \mathrm{Ba} \mathrm{SO} \mathrm{A}_{4}=0,0231 \mathrm{~g} \mathrm{~S}=9,11 \% \mathrm{~S}$.

Berechnet

auf die Formel $\mathrm{C}_{20} \mathrm{H}_{24} \mathrm{~N}_{2} \mathrm{O}_{2} \mathrm{~S}$;

$\mathrm{C}_{20}=240=67,41 \%$

$\mathrm{H}_{24}=24=6,74$,

$\mathrm{N}_{2}=28=7,89$ "

$\mathrm{O}_{2}=32=8,98$,

$\frac{\mathrm{S}=32=8,98 \%}{356100,00 \%}$
Gefunden :

I.

II.

Thiodiglykolsäuredi-p-xylidid

$$
\left[\mathrm{p}: \mathrm{C}_{6} \mathrm{H}_{3}<\underset{\mathrm{NH}}{\mathrm{NH}} . \mathrm{CO} \mathrm{CH}_{2}\right]_{2} \cdot \mathrm{S} \text {. }
$$

Aus alkoholischer Lösung scheidet sich die Verbindung in weissen, feinen Nadeln ab, welche bei $210^{\circ}$ schmelzen. In Alkohol und Essigester sind dieselben schwer löslich, leichter in Eisessig beim Erwärmen, unlöslich in Wasser. 
W. Grothe: Einwirk. จ. Kaliumsulf hydrat etc. auf Chloracetylanilide. 603

\section{Analysen:}

I. $0,193 \mathrm{~g}$ Substanz gaben $0,4787 \mathrm{~g} \mathrm{CO}_{2}=0,1305 \mathrm{~g} \mathrm{C}=67,61 \% \mathrm{C}$ und $0,1218 \mathrm{~g} \mathrm{H}_{2} \mathrm{O}=0,0135 \mathrm{~g} \mathrm{H}=7,01 \% \mathrm{H}$.

II. $0,1635 \mathrm{~g}$ Substanz gaben bei $19^{\circ}$ und $757 \mathrm{~mm}$ Barometerstand $11 \mathrm{ccm}$ feuchten Stickstoff $=7,70 \% \mathrm{~N}$.

III. $0,248 \mathrm{~g}$ Substanz gaben $0,1675 \mathrm{~g} \mathrm{BaSO} \mathrm{A}_{4}=0,023 \mathrm{~g} \mathrm{~S}=9,26 \% \mathrm{~S}$.

Wie die oben angeführten Werte der 0 :Verbindung zeigen, enthält die Formel $\mathrm{C}_{20} \mathrm{H}_{24} \mathrm{~N}_{2} \mathrm{O}_{2} \mathrm{~S} 67,41 \% \mathrm{C}, 6,74 \% \mathrm{HI}, 7,89 \% \mathrm{~N}$ und $8,98 \% \mathrm{~S}$.

Gefunden wurden: I. $67,61 \% \mathrm{C}$ und 7,01\% $\mathrm{H} ;$ II. $7,70 \% \mathrm{~N}$; III. 9,26\% $\mathrm{S}$.

Thiodiglyk olsäuredi-p-phenetidid

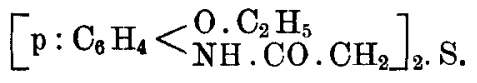

Aus Alkohol oder wenig verdünntem Eisessig erhält man den Körper in schönen weissen, glänzenden Nadeln vom Schmelzpunkte $221^{\circ}$. Sie sind unlöslich in Wasser, schwer löslich in Alkohol, Eisessig, Essigester.

\section{Analysen:}

I. $0,1786 \mathrm{~g}$ Substanz gaben $0,406 \mathrm{~g} \mathrm{CO}_{\mathrm{g}}=0,1107 \mathrm{~g} \mathrm{C}=61,99 \% \mathrm{C}$ und $0,1012 \mathrm{~g} \mathrm{H}_{2} \mathrm{O}=0,01124 \mathrm{~g} \mathrm{H}=6,29 \% \mathrm{H}$.

II. $0,1914 \mathrm{~g}$ Substanz gaben bei $18^{\circ}$ und $753 \mathrm{~mm}$ Barometerstand $12 \mathrm{ccm}$ feuchten Stickstoff $=7,22 \% \mathrm{~N}$.

III. $0,2358 \mathrm{~g}$ Substanz gaben $0,1453 \mathrm{~g} \mathrm{Ba} \mathrm{SO} 4=0,0199 \mathrm{~g} \mathrm{~S}=8,46 \% \mathrm{~S}$.

Berechnet

für die Formel $\mathrm{C}_{20} \mathrm{H}_{24} \mathrm{~N}_{2} \mathrm{O}_{4} \mathrm{~S}$ :

$\mathrm{C}_{20}=240=61,85 \%$

$\mathrm{H}_{24}=24=6,18$,

$N_{2}=28=7,22 n$

$0_{4}=64=16,51$,

$\mathrm{S}=32=8,24$

$388 \quad 100,00 \%$.
Gefunden:

I. II, III.

$61,99 \%$

6,29,

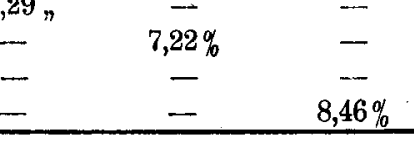

Thiodiglykolsäuredimethylanilid

$$
\left[\mathrm{C}_{6} \mathrm{H}_{5} \mathrm{~N}<\mathrm{CO}_{\mathrm{CH}}^{\mathrm{CH}_{3}}\right]_{2} \cdot \mathrm{S} \text {. }
$$

Bei dieser Verbindung musste, da dieselbe in Alkohol leicht löslich ist, die vom abgeschiedenen Chlorkalium abfiltrierte Lösung eingedunstet werden. Der hinterbleibende, ölige Rückstand wurde mit Wasser innig verarbeitet, bis er fest wurde. Nach mehrmaligem Umkrystallisieren aus stark verdtinntem Alkohol wurden derbe, monokline, glänzende Säulen erhalten, welche bei $115^{\circ}$ schmolzen. Sie sind unlöslich in Wasser, werden aber reichlich von Alkohol, Eisessig, Benzol, Essigester gelöst. 
I. $0,184 \mathrm{~g}$ Substanz gaben $0,4465 \mathrm{~g} \mathrm{CO}_{2}=0,1217 \mathrm{~g} \mathrm{C}=66,14 \% \mathrm{C}$ und $0,1016 \mathrm{~g} \mathrm{H}_{2} \mathrm{O}=0,0113 \mathrm{~g} \mathrm{H}=6,13 \% \mathrm{H}$.

II. 0,2014 g Substanz gaben $0,146 \mathrm{~g} \mathrm{Ba} \mathrm{SO}{ }_{4}=0,020 \mathrm{~g} \mathrm{~S}=9,95 \% \mathrm{~S}$.

Berechnet auf die Formel $\mathrm{C}_{18} \mathrm{H}_{20} \mathrm{~N}_{2} \mathrm{O}_{2} \mathrm{~S}$ :

\begin{tabular}{lccc}
$\mathrm{C}_{18}=216=65,85 \%$ & $66,14 \%$ & - \\
$\mathrm{H}_{20}=20=6,09 \%$ & $6,13 \%$ & - \\
$\mathrm{N}_{2}=28=8,56 \%$ & - & - \\
$\mathrm{O}_{2}=32=9,75 \%$ & - & - \\
$\mathrm{S}=32=9,75 \%$ & - & $9,95 \%$ \\
\hline 328 & $100,00 \%$. &
\end{tabular}

\section{Verhalten der Thiodiglykolsäureanilide gegen Oxydationsmittel.}

Aus den so erbaltenen Sulfiden wurde nun versucht, durch Einwirkung oxydierender Mittel die entsprechenden Sulfone darzustellen. Als bestes Reagenz erwies sich hier das Kaliumpermanganat, und verlief der Prozess in folgender Weise am guinstigsten. Das Sulfid wurde in eizer hinreichenden Menge Eisessig gelöst, und in die fast erkaltete Lösung nach und nach unter fortwährendem Umschütteln eine 6-8 essigsaure Lösung des Permanganats eingetragen, bis eine dauernde Rötung festgestellt wurde. Von Zeit zu Zeit wurde das Gemisch, um einer Abscheidung des schwerlöslichen Sulfides vorzubeugen, kurze Zeit im Wasserbade erhitzt. Nach dem völligen Erkalten wurde dann das Reaktionsprodukt mit wässeriger schwefliger Säure entfärbt, der abgeschiedene, fein verteilte, unlösliche Körper abfiltriert und durch Umkrystallisieren aus einem geeigneten Lösungsmittel gereinigt. Giebt nun auch die beschriebene Methode im allgemeinen den Weg zur Gewinnung dieser Sulfone an, so verläuft der Prozess doch keineswegs immer ganz glatt. Zuweilen kann die Oxydation leicht zu weit gehen, man gewinnt dann weder nach dem Behandeln mit schwefliger Säure, noch nach dem Ausäthern ein Sulfon, sondern es scheint dann eine Zerstörung des Sulfides erfolgt zu sein. Mitunter aber erhält man wohl feste Körper, doch stellen dieselben dann keine einheitlichen Verbindungen dar, es sind Gemische von Sulfid und Sulfon. Ein einheitliches Verfahren, das man, um diese Nachteile auszuschliessen, bei den Oxydationen anzuwenden hat, lässt sich nicht genau angeben, da der einzuschlagende Weg sich in erster Linie nach der grösseren oder geringeren Zersetzlichkeit des zur Oxydation verwendeten Thioäthers richtet.

Für die bei der Oxydation dieser Sulfide entstehenden Sulfone gilt die allgemeine Formel: $(\mathrm{R} \cdot \mathrm{NHCO} \mathrm{CH})_{2} \cdot \mathrm{SO}_{2}$. 
W. Grothe: Einwirk. v. Kaliumsulf hydrat etc. auf Chloracetylanilide.

Sulfondiessigsäureanilid

$\left(\mathrm{C}_{6} \mathrm{H}_{5} \mathrm{NH} \cdot \mathrm{COCH}_{2}\right)_{2} \cdot \mathrm{SO}_{2}$.

Das Sulfon krystallisiert aus seiner reichlich mit Eisessig versetzten, alkoholischen Lösung beim Erkalten in leichten, weissen, rhombischen Blättchen, welche bei $220^{\circ}$ erweichen und bei $225^{\circ}$ unter Zersetzung schmelzen. Es ist unlöslich in Wasser, Benzol, Ligroin, leicht löslich in Eisessig und Essigester, schwer löslich in Alkohol.

Analysen:

I. $0,1718 \mathrm{~g}$ Substanz gaben $0,3656 \mathrm{~g} \mathrm{CO}=0,0997 \mathrm{~g} \mathrm{C}=58,03 \% \mathrm{C}$ und $0,0802 \mathrm{~g} \mathrm{H}_{2} \mathrm{O}=0,0089 \mathrm{~g} \mathrm{H}=\mathbf{5 , 1 2} \% \mathrm{H}$.

II. $0,1624 \mathrm{~g}$ Substanz gaben $0,3474 \mathrm{~g} \mathrm{CO}_{2}=0,0947 \mathrm{~g} \mathrm{C}=58,33 \% \mathrm{C}$ und $0,0746 \mathrm{~g} \mathrm{H}_{2} \mathrm{O}=0,00828 \mathrm{~g} \mathrm{H}=5,10 \% \mathrm{H}$.

III. $0,2534 \mathrm{~g}$ Substanz gaben $0,1823 \mathrm{~g} \mathrm{BaSO}=0,025 \mathrm{~g} \mathrm{~S}=9,80 \% \mathrm{~S}$.

Berechnet

auf die Formel $\mathrm{C}_{16} \mathrm{H}_{16} \mathrm{~N}_{2} \mathrm{SO}_{4}$ :

$$
\mathrm{C}_{16}=192=57,83 \%
$$

$\mathrm{H}_{10}=16=4,84$,

$\mathrm{N}_{2}=28=8,43$,

$\mathrm{S}=32=9,63^{n}$

$0_{4}=64=19,27$,
I. $58,03 \%$ 5,12 Gefunden:

II.

III.

$$
\begin{aligned}
& \text { Sulfondiessigsäure-0-toluidid } \\
& {\left[0: \mathrm{C}_{6} \mathrm{H}_{4}<\mathrm{NH}_{3} \mathrm{CO} \cdot \mathrm{CH}_{2}\right]_{3} \cdot \mathrm{SO}_{2} .}
\end{aligned}
$$

Aus verdünntem Eisessig krystallisiert die Verbindung in weissen, glänzenden, monoklinen Säulen, welche zwischen $225-226^{\circ}$ schmelzen. Wenig löslich ist sie in Alkohol, Methylalkohol, Aether, Essigester, leicht löslich in Eisessig.

\section{Analyse:}

I. $0,1658 \mathrm{~g}$ Substanz gaben $0,365 \mathrm{~g} \mathrm{CO} \mathrm{CO}_{2}=0,09954 \mathrm{~g} \mathrm{C}=60,11 \% \mathrm{C}$ und $0,0841 \mathrm{~g} \mathrm{H}_{2} \mathrm{O}=0,00934 \mathrm{~g} \mathrm{H}=5,64 \% \mathrm{H}$.

\section{Berechnet}

auf die Formel $\mathrm{C}_{18} \mathrm{H}_{20} \mathrm{~N}_{2} \mathrm{~S} \mathrm{O}_{4}$ :

$$
\begin{aligned}
& \mathrm{C}_{18}=216=60,00 \% \\
& \mathrm{H}_{20}=20=5,56 \% \\
& \mathrm{~S}=32=8,88 \% \\
& \mathrm{~N}_{2}=28=7,78 \% \\
& \mathrm{O}_{4}=64=17,78 \% \\
& \hline 360 \quad 100,00 \%
\end{aligned}
$$

Sulfondiessigsäure-p-toluidid

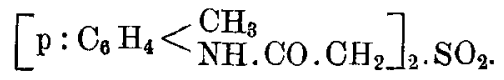

Das braun gefärbte Rohprodukt wird durch Umkrystallisieren aus einer Mischung von verdünntem Eisessig und Alkohol unter Zusatz 
W. Grothe: Einwirk. v. Kaliumsulf hydrat etc. auf Chloracetylanilide.

von Tierkohle gereinigt und bildet kleine, weisse, krystallinische Blättchen vom Schmelzpunkt 2210. Das Sulfon ist unlöslich in Wasser, Ligroin, Benzol, schwer löslich in Alkohol, leichter in Eisessig und Essigester.

\section{Analyse:}

I. $0,167 \mathrm{~g}$ Substanz gaben $0,369 \mathrm{~g} \mathrm{CO}_{2}=0,1006 \mathrm{~g} \mathrm{C}=60,25 \% \mathrm{C}$ und $0,0878 \mathrm{~g} \mathrm{H}_{2} \mathrm{O}=0,00975 \mathrm{~g} \mathrm{H}=5,84 \% \mathrm{H}$.

Analog der Orthoverbindung enthält das p-Derivat von der unitären Formel $\mathrm{C}_{18} \mathrm{H}_{20} \mathrm{~N}_{2} \mathrm{SO}_{4}: \quad 60,00 \% \mathrm{C}, 5,56 \% \mathrm{H}$.

Die Analyse ergab die Werte: $60,25 \% \mathrm{C}$ and $5,84 \% \mathrm{H}$.

$$
\begin{aligned}
& \text { Sulfondiessigsäure-o-xy lidid } \\
& {\left[0: \mathrm{C}_{6} \mathrm{H}_{8}<\mathrm{NH} \text { ( } \mathrm{CH}_{3}\right)_{2}} \\
& {\left[\mathrm{CH}_{2}\right]_{2} \cdot \mathrm{SO}_{2} .}
\end{aligned}
$$

Die Gewinnung dieses Sulfons gelang nicht. Es konnte weder nach der oben angeführten Methode, noch durch Oxydation mit Chromsäure erhalten werden. Ebensowenig führte die Oxydation des in Benzol gelösten Sulfides durch Kaliumpermanganat bei Anwendung eines Rïhrwerkes zum Ziel. Meist wurden gar keine Ausbenten erhalten; eine geringe im Schmelzpunkt vom Sulfid abweichende Menge erwies sich bei der Analyse als grösstenteils noch aus Sulfid bestehend. Der Grund hierfür liegt wahrscheinlich in der geringen Beständigkeit des enstandenen Sulfons.

$$
\begin{aligned}
& \text { Sulfondiessigsäure-p-xylidid } \\
& {\left[\mathrm{p}: \mathrm{C}_{6} \mathrm{H}_{3}<\mathrm{NH} \text {. } \mathrm{CO} . \mathrm{CH}_{2}\right]_{2} \cdot \mathrm{SO}_{2} \text {. }}
\end{aligned}
$$

Das Sulfon scheidet sich aus seiner Lösung in Eisessig in weissen, dünnen, sehr leichten Nadeln ab, welche bei $237^{\circ}$ schmelzen. Dieselben sind in den meisten Lösungsmitteln wenig oder nicht löslich, reichlicher in Eisessig.

\section{Analyse:}

I. $0,1753 \mathrm{~g}$ Substanz gaben $0,3983 \mathrm{~g} \mathrm{CO}_{\mathrm{a}}=0,1087 \mathrm{~g} \mathrm{C}=61,96 \% \mathrm{C}$ und $0,0988 \mathrm{~g} \mathrm{H}_{2} \mathrm{O}=0,0109 \mathrm{~g} \mathrm{H}=6,26 \% \mathrm{H}$.

Berechnet

auf die Formel $p: \mathrm{C}_{20} \mathrm{H}_{24} \mathrm{~N}_{2} \mathrm{~S} \mathrm{O}_{4}$ :

Gefunden:

$$
\begin{array}{lr}
\mathrm{C}_{20}=240=61,85 \% & 61,96 \% \\
\mathrm{H}_{24}=24=6,19 n & 6,26 \% \\
\mathrm{~N}_{2}=28=6,26^{\prime} & - \\
\mathrm{S}=32=8,24_{n} & - \\
\mathrm{O}_{4}=64=16,48 . & - \\
\hline 388 \quad 100,00 \% . &
\end{array}
$$

I. 
W. Grothe: Einwirk. v. Kaliumsulf hydrat etc. auf Chloracetylanilide.

607

Sulfondiessigsäure-p-phenetidid

$$
\left[\mathrm{p}: \mathrm{C}_{6} \mathrm{H}_{4}<\underset{\mathrm{NH}}{\mathrm{NH}} \mathrm{OCOH}_{2}\right]_{2} \cdot \mathrm{SO}_{2} \text {. }
$$

Aus verdünntem Eisessig krystallisiert das Sulfon in Form rhombischer Blättchen, welche bei $239^{\circ}$ schmelzen. Dieselben sind schwer löslich in Alkohol, Aether, Benzol, Ligroin, leichter in Eisessig und Essigester.

Analyse:

I. $0,1533 \mathrm{~g}$ Substanz gaben $0,3223 \mathrm{~g} \mathrm{CO}_{2}=0,0879 \mathrm{~g} \mathrm{C}=57,33 \% \mathrm{C}$ und $0,081 \mathrm{~g} \mathrm{H}_{2} \mathrm{O}=0,009 \mathrm{~g} \cdot \mathrm{H}=5,87 \% \mathrm{H}$.

$$
\text { Berechnet }
$$

auf die Formel $\mathrm{C}_{20} \mathrm{H}_{24} \mathrm{~N}_{2} \mathrm{SO}_{6}$ :

Gefunden:

\begin{tabular}{lc}
$\mathrm{C}_{20}=240=57,11 \%$ & $57,33 \%$ \\
$\mathrm{H}_{24}=24=5,78 \%$ & 5,87 \\
$\mathrm{~N}_{2}=28=6,70 \%$ & - \\
$\mathrm{S}=32=7,65 \%$ & - \\
$\mathrm{O}_{6}=96=22,76 \%$ & - \\
\hline $420 \quad 100,00 \%$. &
\end{tabular}

Sulfondiessigsäuremethylanilid

$$
\left[\mathrm{C}_{6} \mathrm{H}_{5} . \mathrm{N}<\underset{\mathrm{CO} . \mathrm{CH}_{2}}{\mathrm{CH}_{2}} \cdot \mathrm{SO}_{2}\right. \text {. }
$$

Aus verdünntem Eisessig scheidet sich die Verbindung in Form schöner, glänzender, farbloser Nadeln ab, welche bei $152^{\circ}$ schmelzen. In Wasser, Aether und Benzol löst sich das Sulfon gar nicht oder nur wenig, wird aber von Essigester, Alkohol und Eisessig reichlich aufgenommen.

Analysen:

I. $0,1798 \mathrm{~g}$ Substanz gaben $0,3954 \mathrm{~g} \mathrm{CO}_{2}=0,1078 \mathrm{~g} \mathrm{C}=59,97 \% \mathrm{C}$ und $0,0929 \mathrm{~g} \mathrm{H}_{2} \mathrm{O}=0,0103 \mathrm{~g} \mathrm{H}=5,74 \% \mathrm{H}$.

II. $0,2858 \mathrm{~g}$ Substanz gaben $0,1826 \mathrm{~g} \mathrm{BaSO}_{4}=0,02507 \mathrm{~g} \mathrm{~S}=8,77 \% \mathrm{~S}$.

Wie bei den isomeren Verbindungen der beiden Toluidine ausführlich festgestellt, verlangt das Sulfon der Formel $\mathrm{C}_{18} \mathrm{H}_{20} \mathrm{~N}_{2} \mathrm{SO}_{4}: 60,00 \% \mathrm{C}, 5,56 \% \mathrm{H}$, $8,88 \% \mathrm{~S}$.

Gefunden: I. $59,97 \% \mathrm{C}, 5,74 \% \mathrm{H}$; II. $8,77 \% \mathrm{~S}$.

\section{Einwirkung von Cyankalium auf die chloracetylierten Anilide.}

Es wurde nun weiter versucht, das Chloratom gegen die Cyangruppe auszutauschen, um so Verbindungen der allgemeinen Formel R.NH.CO. $\mathrm{CH}_{2} \cdot \mathrm{CN}$ zu erhalten. $\mathrm{Zu}$ diesem Zwecke wurde die alkoholische Lösung der Chloracetylverbindungen mit einer wässerig alkoholischen Lösung von Cyankalium in geringem Ueberschuss im Wasserbade erhitzt. Nach Beendigung der Reaktion wurde vom Chlorkalium abfiltriert, das Filtrat noch etwas mit Wasser verdünnt und zur Krystallisation gestellt. Die ausgeschiedenen Krystalle wurden durch Umkrystallisieren gereinigt. Nach diesem Verfahren gelang es 
jedoch nicht beim 0 -Toluidin, 0 -Xylidin, $p$-Phenetidin die gewünschten Cyanverbindungen zu erhalten. Die vom Chlorkalium abfiltrierte Lösung schied keine Krystalle ab, auch nicht nach weiterem Verdünnen mit Wasser. Versucht man durch Eindunsten derselben die Körper abzuscheiden, so resultieren tiefrotbraun gefärbte, schmierige Rückstände, die auf keine Weise zu reinigen waren, da die schmierigen Zersetzungsprodukte in alle Lösungsmittel eingehen.

Erhalten wurde das schon von $Q u$ enda ${ }^{1}$ ) durch Einwirkung von Cyanessigester auf Anilin gewonnene Cyanacetanilid vom Schmelzpunkt $198,5-200^{\circ}$ und das in gleicher Weise von Guareschi ${ }^{2}$ ) dargestellte Cyanacetmonomethylanilid vom Schmelzpunkt $86-87,5^{\circ}$. Beide Verbindungen wurden durch Vergleichung ihrer physikalischen Eigenschaften und Feststellung ihres Schmelzpunktes identifiziert. Dargestellt wurden ferner die Cyanacetylverbindungen des $\mathbf{p}$-Toluidins und $\mathrm{p}$ - Xylidins.

$$
\begin{gathered}
\text { Cyanacet-p-toluidid } \\
\text { p: } \mathrm{C}_{6} \mathrm{H}_{4}<\underset{\mathrm{NH}}{\mathrm{CH}_{3} \mathrm{CO} . \mathrm{CH}_{2} \mathrm{CN} .}
\end{gathered}
$$

Der Körper krystallisiert aus der verdünnten alkoholischen Lösung in feinen, glänzend weissen, monoklinen Blättchen vom Schmelzpunkt $180^{\circ}$. Dieselben sind unlöslich in Wasser und Aether, schwer löslich in Ligroin, leicht löslich in den übrigen Lösungsmitteln.

\section{Analysen:}

I. $0,1678 \mathrm{~g}$ Substanz gaben $0,4263 \mathrm{~g} \mathrm{CO}=0,1162 \mathrm{~g} \mathrm{C}=69,28 \% \mathrm{C}$ und $0,0896 \mathrm{~g} \mathrm{H}_{2} \mathrm{O}=0,00965 \mathrm{~g} \mathrm{H}=5,75 \% \mathrm{H}$.

II. $0,1983 \mathrm{~g}$ Substanz gaben bei $758 \mathrm{~mm}$ Barometerstand und $20^{\circ} \mathrm{C}$. $28,3 \mathrm{ccm}$ feuchten Stickstoff $=16,28 \% \mathrm{~N}$.

\section{Berechnet}

auf die Formel $\mathrm{C}_{10} \mathrm{H}_{10} \mathrm{~N}_{2} \mathrm{O}$ :

$$
\mathrm{C}_{10}=120=68,95 \%
$$

$\mathrm{H}_{10}=10=5,74$,

$\mathrm{N}_{2}=28=16,09_{n}$

$0=16=9,22$,
Gefunden:

I.

II.

$174100,00 \%$.

Cyanacet-p-xylidid

$$
\mathrm{p}: \mathrm{C}_{6} \mathrm{H}_{3}<\underset{\mathrm{NH}}{\mathrm{NH}} \stackrel{\left(\mathrm{CH}_{3}\right)_{2}}{\mathrm{CO}} \mathrm{CH}_{2} \mathrm{CN} \text {. }
$$

Aus Alkohol scheidet sich der Körper in wohl ausgebildeten, glänzenden farblosen Nadeln $\mathrm{ab}$, welche bei $167^{\circ}$ schmelzen. Dieselben sind unlöslich in Wasser, löslich in den übrigen Lösungsmitteln.

1) Beilstein II (Privatm.) 363.

2) Ebenda (Privatm.) 366. 
W. Grothe: Einwirk. v. Kaliumsulf hydrat etc. auf Chloracetylanilide. 609

$$
\text { Analysen: }
$$

I. $0,1868 \mathrm{~g}$ Substanz gaben $0,4802 \mathrm{~g} \mathrm{CO}_{2}=0,1309 \mathrm{~g} \mathrm{C}=70,10 \% \mathrm{C}$ und $0,111 \mathrm{~g} \mathrm{H}_{2} \mathrm{O}=0,01237 \mathrm{~g} \mathrm{H}=6,62 \% \mathrm{H}$.

II. $0,1682 \mathrm{~g}$ Substanz gaben bei $757 \mathrm{~mm}$ Barometerstand und $22^{\circ} \mathrm{C}$. $21,8 \mathrm{ccm}$ feuchten Stickstoff $=14,60 \% \mathrm{~N}$.

\section{Berechnet}

auf die Formel $\mathrm{C}_{11} \mathrm{H}_{13} \mathrm{~N}_{2} \mathrm{O}$ :

$$
\begin{aligned}
& \mathrm{C}_{11}=132=70,21 \% \\
& \mathrm{H}_{12}=12=6,39 \% \\
& \mathrm{~N}_{2}=28=14,89 \% \\
& \mathrm{O}=16=8,51 \% \\
& \hline 188100,00 \%
\end{aligned}
$$

\section{Versuche, aus den Cyanacetylverbindungen durch Verseifung die zugehörigen Säuren darzustellen.}

Es wurde nun versucht, in den dargestellten Cyanverbindungen die Cyangruppe zu verseifen, um so zu Säuren der allgemeinen Formel

$$
\text { R. NH . CO . } \mathrm{CH}_{2} \mathrm{COOH}
$$

zu gelangen. $Z u$ diesem $Z$ wecke wurde die betr. Cyanverbindung mit konzentrierter Salzsäure mehrere Stunden lang im geschlossenen Rohr auf ca. $140^{\circ}$ erhitzt. Nach dieser Zeit zeigte der Rohrinhalt reichliche Abscheidung von nadelförmigen Krystallen und beim Oeffnen des Rohrs wurde eine heftige Gasentwickelung beobachtet. Die abfiltrierten Krystalle waren in Wasser leicht löslich und wurden durch geeignete Reaktionen als salzsaures Salz des in Frage stehenden Amins erkannt. Weitere Mengen desselben krystallisierten beim Eindampfen der salzsauren Lösung aus, welche nach dem Abfiltrieren derselben mit kohlensaurem Natrium neutralisiert und zur Trockne verdampft wurde. Im Rïckstand liess sich sowohl Essigsäure wie Ammoniak nachweisen. Der diesen Thatsachen zu Grunde liegende chemische Prozess ist folgender:

$$
\begin{aligned}
& \text { R. NH } . \mathrm{COCH}_{2} \mathrm{CN}+2 \mathrm{HCl}+3 \mathrm{H}_{2} \mathrm{O}= \\
& \text { R. } \mathrm{NH}_{2} \cdot \mathrm{HCl}+\mathrm{CH}_{2}<\mathrm{COOH}+\mathrm{NH}_{4} \mathrm{Cl} .
\end{aligned}
$$

Es wurde also der ganze Acetylrest abgespalten und zu Malonsăure verseift unter Bildung des salzsauren Amins. Die entstandene Malonsäure aber zerfällt schon bei einer $132^{\circ}$ übersteigenden Temperatur glatt in Essigsäure und Kohlendioxyd.

$$
\mathrm{CH}_{2}<\underset{\mathrm{COOH}}{\mathrm{COOH}}=\mathrm{CO}_{2}+\mathrm{CH}_{3} . \mathrm{COOH} \text {. }
$$

Es konnte daher später nur Essigsäure nachgewiesen werden und erklärt sich aus diesem Zerfall der Malonsäure die schon erwähnte 
lebhafte Gasentwickelung beim Oeffnen des Rohres. Verschiedene andere unter gewöhnlichem Druck mit verdünnnter Salzsäure oder Schwefelsäure angestellte Versuche führten ebenfalls zu keinem positiven Ergebnis, sondern verliefen in gleicher Weise unter Zersetzung des Ausgangsmaterials.

\section{Einwirkung von Rhodankalium auf die Chloracetylanilide.}

Um das Verhalten der Chloracetylverbindungen gegen Rhodankalium zu prüfen, wurden die alkoholischen Lösungen derselben mit alkoholischer Rhodankaliumlösung etwa eine halbe Stunde lang im Wasserbade erbitzt. Die rom abgeschiedenen Chlorkalium abfiltrierte Lösung wurde dann entweder zur Krystallisation gestellt oder, falls keine Abscheidung eines krystallinischen Körpers erfolgte, auf ein kleines Volumen eingedampft, und mit kaltem Wasser der Körper abgeschieden. Durch Umkrystallisieren aus geeigneten Lösungsmitteln teilweise nnter Zusatz von Tierkohle gelang es bei allen Chloracetaniliden Rhodanacetylverbindungen in reinem Zustande darzustellen, deren Bildung die folgende allgemein giltige Gleichung veranschanlicht:

$\mathrm{R} . \mathrm{NH} . \mathrm{COCH}_{2} \mathrm{Cl}+\mathrm{KSCN}=\mathrm{KCl}+\mathrm{RNH} . \mathrm{CO} . \mathrm{CH}_{2} \mathrm{SCN}$.

$$
\begin{gathered}
\text { Rhodanacetanilid } \\
\mathrm{C}_{6} \mathrm{H}_{5} \cdot \mathrm{NH} \cdot \mathrm{CO} \cdot \mathrm{CH}_{2} \cdot \mathrm{SCN} .
\end{gathered}
$$

Durch Abscheidung aus der konzentrierten alkoholischen Lösung mit Wasser erhält man den Körper in Form feiner, glänzend weisser Nädelchen, welche zwischen $86-87^{\circ}$ schmelzen. Dieselben sind nicht löslich in kaltem, schwer in heissem Wasser, leicht dagegen in Aethylalkohol, Benzol, Eisessig, Essigester.

Analysen:

I. $0,1708 \mathrm{~g}$ Substanz gaben $0,3524 \mathrm{~g} \mathrm{CO}_{2}=0,0961 \mathrm{~g} \mathrm{C}=56,26 \% \mathrm{C}$ und $0,068 \mathrm{~g} \mathrm{H}_{8} \mathrm{O}=0,0075 \mathrm{~g} \mathrm{H}=4,42 \underset{\%}{o} \mathrm{H}$.

II. $0,1879 \mathrm{~g}$ Substanz gaben bei $750 \mathrm{~mm}$ Barometerstand and $24^{\circ} \mathrm{C}$. $25 \mathrm{ccm}$ feuchten Stickstoff $=18,86 \% \mathrm{~N}$.

III. $0,1759 \mathrm{~g}$ Substanz gaben $0,2174 \mathrm{~g} \mathrm{Ba} \mathrm{SO}_{4}=0,0298 \mathrm{~g} \mathrm{~S}=16,96 \%$.

Berechnet

auf die Formel $\mathrm{C}_{8} \mathrm{H}_{8} \mathrm{~N}_{2} \mathrm{SO}$ :

$\mathrm{C}_{9}=108=56,25 \%$

$\mathrm{H}_{8}=8=4,18$,

$\mathrm{N}_{2}=28=14,58$,

$0=16=8,33$ ",

$\mathrm{S}=32=16,66$.
Gefunden:

I.

II.

III.

\begin{tabular}{rrrrr}
$\mathrm{C}_{9}=108=56,25 \%$ & $56,26 \%$ & - & - \\
$\mathrm{H}_{8}=8=4,18 \%$ & $4,42 \%$ & - & - \\
$\mathrm{N}_{2}=28=14,58 \%$ & - & $14,86 \%$ & - \\
$0=16=8,33 \%$ & - & - & - \\
$\mathrm{S}=32=16,66 \%$ & - & - & $16,96 \%$ \\
\hline 192 & $100,00 \%$ &
\end{tabular}


W. Grothe: Einwirk. v. Kaliumsulfhydrat etc. auf Chloracetylanilide. 611

$$
\begin{gathered}
\text { Rhodanacet-0-toluidid } \\
\text { o: } \mathrm{C}_{6} \mathrm{H}_{4}<\mathrm{CH}_{3} \text {. CO.CH } . \mathrm{SCN} \text {. }
\end{gathered}
$$

Aus verdünnter alkoholischer Lösung krystallisiert die Verbindung in sehr feinen, weissen Nadeln vom Schmelzpunkt $102-103^{\circ}$. Dieselben sind wenjg löslich in Wasser, löslich in den meisten übrigen Lösungsmitteln. Analysen:

I. $0,2035 \mathrm{~g}$ Substanz gaben bei $750 \mathrm{~mm}$ Barometerstand und $250,24,5 \mathrm{ccm}$ feuchten Stickstoff $=13,24 \% \mathrm{~N}$.

II. $0,2258 \mathrm{~g}$ Substanz gaben $0,2492 \mathrm{~g} \mathrm{BaSO}_{4}=0,0342 \mathrm{~g} \mathrm{~S}=15,15 \% \mathrm{~S}$. Berechnet auf die Formel $\mathrm{C}_{10} \mathrm{H}_{10} \mathrm{~N}_{2} \mathrm{SO}$ :

I.

Gefunden:

$$
\begin{aligned}
& \mathrm{C}_{10}=120=58,25 \% \\
& \mathrm{H}_{10}=10=4,85 \% \\
& \mathrm{~N}_{2}=28=13,59 \% \\
& \mathrm{~S}=32=15,55 \% \\
& \mathrm{O}=16=7,76 \%
\end{aligned}
$$

Rhodanacet-p-toluidid

$$
p: \mathrm{C}_{6} \mathrm{H}_{4}<\mathrm{NH}_{3} \mathrm{CHOCH}_{2} . \mathrm{SCN} \text {. }
$$

Man erhält diesen Körper aus sehr verdünnter alkoholischer Lösung in feinen, schwach gefärbten, bei $125-126^{\circ}$ schmelzenden Nadeln, welche in heissem Wasser und den übrigen Lösungsmitteln reichlich löslich sind.

\section{Analysen:}

I. 0,2234 g Substanz gaben bei $23^{\circ}, 752 \mathrm{~mm}$ Barometerstand $27,3 \mathrm{ccm}$ feuchten Stickstoff $=13,62 \% \mathrm{~N}$.

II. $0,2682 \mathrm{~g}$ Substanz gaben $0,3052 \mathrm{~g} \mathrm{BaSO} \mathrm{Ba}_{4}=0,0419 \mathrm{~g} \mathrm{~S}=15,62 \% \mathrm{~S}$.

Analog den oben ausführlich erwähnten Werten der 0 :Verbindung enthält die Formel $\mathrm{C}_{10} \mathrm{H}_{10} \mathrm{~N}_{2} \mathrm{SO} 13,59 \% \mathrm{~N}$ und $15,55 \% \mathrm{~S}$.

Gefunden wurde: I. $13,62 \% \mathrm{~N}$ und II. $15,62 \% \mathrm{~S}$.

$$
\begin{gathered}
\text { Rhodanacet-o( } \alpha) \cdot x y l i d i d \\
o(\alpha): \mathrm{C}_{6} \mathrm{H}_{3}<\mathrm{NH} . \mathrm{CO} . \mathrm{CH}_{2} \mathrm{SCN} \text {. }
\end{gathered}
$$

Durch Ausfällen der mit Tierkohle entfärbten, eingeengten alkoholischen Lösung erhält man das Rhodanacetorthoxylidid als weisse, rhombische Blättchen, welche bei $102^{\circ}$ schmelzen. In Wasser und Ligroin sind dieselben. wenig löslich, leicht dagegen in Aether, Alkohol, Eisessig, Benzol.

\section{Analysen:}

I. $0,1914 \mathrm{~g}$ Substanz gaben bei $753 \mathrm{~mm}$ Barometerstand und $25^{\circ} \mathrm{C}$. $22 \mathrm{ccm}$ feuchten Stickstoff $=12,83 \% \mathrm{~N}$.

II. $0,2058 \mathrm{~g}$ Substanz gaben $0,2227 \mathrm{~g} \mathrm{Ba} \mathrm{SO} \mathrm{H}_{4}=0,03058 \mathrm{~g} \mathrm{~S}=14,86 \% \mathrm{~S}$. 
612 W. Grothe: Einwirk. v. Kaliumsulf hydrat etc. auf Chloracetylanilide

Berechnet

auf die Formel $\mathrm{C}_{11} \mathrm{H}_{12} \mathrm{~N}_{2} \mathrm{SO}$ :

$$
\mathrm{C}_{11}=132=60,00 \%
$$

$\mathrm{H}_{12}=12=5,45$ "

$\mathrm{N}_{2}=28=12,74$,

$0=16=7,27$,

$\mathrm{S}=32=14,54$,
Gefunden:

I. II.

11.

$$
220 \quad 100,00 \% \text {. }
$$

Rhodanacet - p-xylidid

$$
\mathrm{p}: \mathrm{C}_{6} \mathrm{H}_{3}<\mathrm{NH} \cdot \mathrm{COCH}_{2} \cdot \mathrm{SCN} \text {. }
$$

Die Verbindung krystallisiert aus Alkohol in weissen, feinen verfilzten Nadeln, welche bei $133^{\circ}$ schmelzen. Dieselben sind schwer löslich in Aether und Ligroin, leicht löslich in Alkohol, Eisessig, Essigester.

\section{Analysen:}

I. $0,2427 \mathrm{~g}$ Substanz gaben bei $760 \mathrm{~mm}$ Druck und $23^{\circ}$ C. $27,3 \mathrm{ccm}$ feuchten Stickstoff $=12,67 \% \mathrm{~N}$.

II. $0,206 \mathrm{~g}$ Substanz gaben $0,2242 \mathrm{~g} \mathrm{BaSO}_{4}=0,03079 \mathrm{~g} \mathrm{~S}=14,94 \% \mathrm{~S}$.

Nach den Berechnungen bei der 0 :Verbindung enthält die Formel $\mathrm{p}: \mathrm{C}_{11} \mathrm{H}_{12} \mathrm{~N}_{2} \mathrm{SO} 12,74 \% \mathrm{~N}$ und $14,54 \% \mathrm{~S}$.

Die Analyse ergab: I. $12,67 \% \mathrm{~N}$ und II. $14,94 \% \mathrm{~S}$.

$$
\begin{gathered}
\mathrm{Rhodanacet} \cdot \mathrm{p} \cdot \mathrm{phenetidid} \\
\mathrm{C}_{6} \mathrm{H}_{4}<\mathrm{NH} \cdot \mathrm{OCOCH}_{2} \mathrm{H}_{5} \mathrm{SCN} .
\end{gathered}
$$

Die Verbindung gewinnt man nach dem Ausfällen mit Wasser durch Umkrystallisieren aus sehr verdünntem Eisessig in schwach gelblich gefärbten, büscheligen Krystallen, welche zwischen $164-165^{0}$ schmelzen. Dieselben sind unlöslich in Aether und Ligroin, schwer löslich in heissem Wasser, leicht löslich in Alkohol, Benzol, Eisessig, Essigester.

\section{Analysen:}

I. $0,2172 \mathrm{~g}$ Substanz gaben bei $759 \mathrm{~mm}$ Barometerstand und $25^{\circ} \mathrm{C}$. $22 \mathrm{ccm}$ feuchten Stickstoff $=11,68 \% \mathrm{~N}$.

II. 0,1937 g Substanz gaben $0,1988 \mathrm{~g} \mathrm{Ba} \mathrm{SO}_{4}=0,0273 \mathrm{~g} \mathrm{~S}=13,73 \% \mathrm{~S}$.

\section{Berechnet}

auf die Formel $\mathrm{C}_{11} \mathrm{H}_{12} \mathrm{O}_{2} \mathrm{NS}$ :

$$
\begin{aligned}
& \mathrm{C}_{11}=132=55,93 \% \\
& \mathrm{H}_{12}=12=5,08 \% \\
& \mathrm{O}_{2}=32=13,55 \% \\
& \mathrm{~N}_{2}=28=11,89 \% \\
& \mathrm{~S}=32=13,55 \% \\
& \hline 236 \quad 100,00 \% .
\end{aligned}
$$

Gefunden:

I.

II.

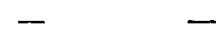


W. Gr othe: Einwirk. v. Kaliumsulfhydrat etc. anf Chloracetylanilide. 613

$$
\begin{gathered}
\text { Rhodanacetmethylanilid } \\
\mathrm{C}_{6} \mathrm{H}_{5} \mathrm{~N}<\mathrm{CO} \cdot \mathrm{CH}_{3} \mathrm{SCN} .
\end{gathered}
$$

Das Rhodanacetmethylanilid krystallisiert aus verdünntem Alkohol in derben, weissen, monoklinen Säulen. Ihr Schmelzpunkt liegt bei $69^{\circ}$. In Wasser sind dieselben nur wenig löslich, leicht aber in Alkohol, Aether, Eisessig, Benzol, Essigester.

\section{Analysen:}

I. 0,2160 g Substanz gaben bei $21^{0}, 752 \mathrm{~mm}$ Barometerstand, $26 \mathrm{ccm}$ feuchten Stickstoff $=13,55 \% \mathrm{~N}$.

II. $0,2054 \mathrm{~g}$ Substanz gaben $0,2365 \mathrm{~g} \mathrm{Ba} \mathrm{SO}_{4}=0,03248 \mathrm{~g} \mathrm{~S}=15,81 \% \mathrm{~S}$.

$$
\text { Berechnet }
$$

auf die Formel $\mathrm{C}_{10} \mathrm{H}_{10} \mathrm{~N}_{2} \mathrm{SO}$ :

Gefunden:

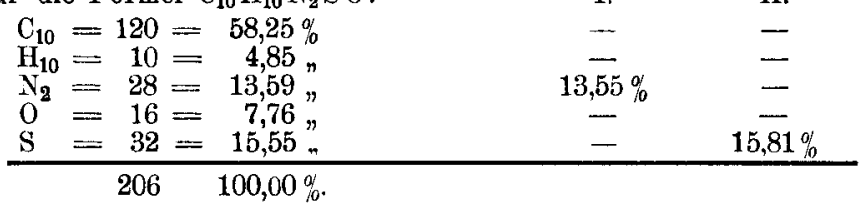

\section{Zusammenstellung der Untersuchungs-Resultate.}

I. Als Ausgangsmaterial dienten folgende chloracetylierten Anilide: Chloracetanilid . . . . . . . . . . Schmelzpunkt $134^{0}$ Chloracet-o-toluidid . . . . . . . . " $\quad 111^{0}$ Chloracet-p-toluidid . . . . . . . . $\quad " 164^{0}$ Chloracet-0-( $\alpha)$-xylidid . . . . . . " $109^{\circ}$ Chloracet-p-xylidid . . . . . . . . " Chloracet-p-phenetidid . . . . . . . ” $148^{\circ}$ Chloracetmethylanilid . . . . . . . " $70^{\circ}$

II. Durch Einwirkung von benzol- und p-tolnolsulfinsaurem Salz auf die Chloracetylderivate wurden erhalten:

Phenylsulfonacetanilid . . . . . . . Schmelzpunkt $142^{0}$ p - Tolylsulfonacetanilid . . . . . . . " " $168^{\circ}$ Phenylsulfonacet-0-toluidid . . . . . " $\quad 150^{\circ}$ p-Tolylsulfonacet-o-toluidid . . . . . $\quad " \quad 129^{\circ}$ Phenylsulfonacet-p-toluidid . . . . . $\quad " \quad 153^{0}$ p-Tolylsulfonacet-p-toluidid . . . . . " " $157^{\circ}$ Phenylsulfonacet- $0-(\alpha)$-xylidid . . . . . " $\quad 141^{\circ}$ $\mathrm{p}$ - Tolylsulfonacet-0-( $\alpha)$-xylidid . . . . $\quad$. $153-154^{0}$ Phenylsulfonacet-p-xylidid . . . . . . " n $143^{\circ}$ p - Tolylsulfonacet-p-xylidid . . . . . . $\quad " 160^{\circ}$ Phenylsulfonacet-p-phenetidid . . . . . " $\quad 151^{0}$ p-Tolylsulfonacet-p-phenetidid. . . . . " " $156^{0}$ 
614 W. Grothe: Einwirk. v. Kaliumsulf hydrat etc, auf Chloracetylanilide.

Phenylsulfonacetmethylanilid . . . . . " $125^{0}$

p-Tolylsulfonacetmethylanilid . . . . . " $112^{0}$

III. Durch alkoholisches Kalihydrat wurden die Phenyl- und Tolylsulfonacetylderivate gespalten in das freie Amin und das entsprechende Sulfon:

Methylphenylsulfon . . . . . . Schmelzpunkt $88^{\circ}$

Methyl-p-Tolylsulfon . . . . . . . . " $86-87^{\circ}$

IV. Bei Behandlung der Chloracetylderivate mit alkoholischem Kaliumsulf hydrat entstanden die Anilide der Thiodiglycolsäure:

Thiodiglycolsäuredianilid . . . . . Schmelzpunkt $169^{\circ}$

Thiodiglycolsänredi-0-toluidid . . . . " $190^{\circ}$

Thiodiglycolsäuredi-p-toluidid . . . . " $194^{0}$

Thiodiglycolsäuredi-o- $(\alpha)$-xylidid . . . . " $\quad 194^{0}$

Thiodiglycolsäuredi-p-xylidid . . . . . , $210^{\circ}$

Thiodiglykolsäuredi-p-phenetidid . . . . " " $221^{0}$

Thiodiglycolsäuredimethylanilid . . . . " $115^{\circ}$

V. Aus den dargestellten Sulfiden konnten durch Oxydation mit Kaliumpermanganat folgende Sulfone erhalten werden:

Sulfondiessigsäureanilid . . . . . . . Schmpkt. 220-2250

Sulfondiessigsäure-0-toluidid . . . . . " $225-226^{\circ}$

Sulfondiessigsäure-p-toluidid . . . . . " " $221^{\circ}$

Sulfondiessigsäure-p-xylidid . . . . . " " . $237^{0}$

Sulfondiessigsäure-p-phenetidid . . . . , " $239^{\circ}$

Sulfondiessigsäuremethylanilid . . . . " " $152^{\circ}$

VI. Beim Behandeln der Chloracetanilide mit Cyankalium entstanden:

Cyanacetanilid . . . . . . . . . Schmpkt. 198,5-200

Cyanacetmetbylanilid . . . . . . . " $86-87,5^{\circ}$

Cyanacet-p-toluidid . . . . . . . . "

Cyanacet-p-xylidid . . . . . . . . " " $167^{\circ}$

VII. Mit Rhodankalium setzten sich die Chloracetylanilide um zu:

Rhodanacetanilid . . . . . . . Schmelzpkt. $86-87^{\circ}$

Rhodanacet-0-toluidid . . . . . . . " 102-1030

Rhodanacet-p-toluidid . . . . . . . ” $125^{\circ}$

Rhodanacet-0-( $\alpha)$-xylidid . . . . . . " " $102^{0}$

Rhodanacet-p-xylidid . . . . . . . " $133^{\circ}$

Rhodanacet-p-phenetidid . . . . . " $164-165^{\circ}$

Rhodanacetmethylaniiid . . . . . . " $\quad 69^{\circ}$

Nicht isoliert konnten die Cyanacetylverbindungen des 0-Toluidins, (a) 0 -Xylidins und des $\mathrm{p}$-Phenetidins werden. Die Oxydation der dargestellten Sulfide zu den entsprechenden Sulfonen führte nur bei der Verbindung des $(\alpha)_{0}$-Xylidins zu keinem befriedigenden Ergebniss. 